\title{
Conditional extensions of fuzzy preorders
}

\author{
J. C. R. Alcantud ${ }^{1}$ and S. Díaz ${ }^{2}$ \\ 1 Facultad de Economía y Empresa and Multidisciplinary Institute of Enterprise \\ (IME) \\ Campus Miguel de Unamuno \\ Universidad de Salamanca, Spain \\ http://diarium.usal.es/jcr \\ jcr@usal.es \\ 2 Dept. of Statistics and O. R., University of Oviedo \\ Calvo Sotelo s/n, 33007 Oviedo, Spain \\ http://unimode.uniovi.es/ \\ diazsusana@uniovi.es
}

\begin{abstract}
The problem of embedding incomplete into complete relations has been an important topic of research in the context of crisp relations and their applications. Several variations of the acclaimed Szpilrajn's theorem have been provided, inclusive of the case when some order conditions between elements are imposed on the extension. We extend the analysis of that topic by Alcantud to the fuzzy case. By appealing to generators to decompose (fuzzy) preference relations into strict preference and indifference relations, we give general extension results for the corresponding concept of compatible extension of a fuzzy reflexive relation. Then we investigate the conditions under which compatible order extensions exist such that certain elements are connected by the asymmetric part, resp., and certain other elements by the symmetric part, to respective elements with degree 1.
\end{abstract}

Keywords: fuzzy relation, transitivity, acyclicity, consistency, extension, (indifference) generator.

\section{Introduction}

As Herden and Pallack [30] put it, "one of the best known theorems in order theory, mathematical logic, computer sciences and mathematical social sciences is the Szpilrajn Theorem which states that every partial order can be refined to a linear order". Another form of the same principle states that any preorder has an order extension (cf., Arrow [5, Chapter VI], Hansson [29, Lemma 3]). Many variations and generalizations followed. Dushnik and Miller [20] prove that any partial order is the intersection of linear orders, which is obtained as a special case of a general extension theorem by Duggan [19]. Donaldson and Weymark [17] (see also Bossert [10]) prove the corresponding result for preorders, namely, that any preorder is the intersection of orders. Suzumura [44], [45, Theorem $\mathrm{A}(5)]$ shows that a property called consistency is necessary and sufficient for 
the existence of an order extension. Alcantud [4] systematizes the identification of constraints that can be imposed on the order extensions. This is important because Szpilrajn's theorem [47] is not constructive thus the researcher cannot proceed by direct inspection of the resulting orders. ${ }^{3}$ Another general scheme for extending Szpilrajn's theorem consists of restricting attention to preorders with some prescribed property, and requiring that their extensions also possess that property. In this line of inspection Foldes and Szigeti [24] give a complete description of the so-called maximal compatible partial orders on $A$ of the arbitrary unary operation $f: A \rightarrow A$, and Mabrouk [36] proves that for any preorder on a real vector space that verifies the property of translation invariance, there is an order that extends it and verifies translation invariance. Bosi and Herden [8, 9], Bossert et al. [11], Campión et al. [14], Herden and Pallack [30], Jaffray [32], or Yi [49] among others discuss continuity and semicontinuity issues in relation with the Szpilrajn theorem. Particularly fruitful is Yi's approach. According to Campión et al. [14], a topological space verifies Yi's extension property -which is more restrictive than topological normality as a separation axiom- when any closed preorder on any closed subset of the space has a continuous extension to the whole space. These authors prove that even a stronger version of its semicontinuous analogue is true irrespective of the topology.

Results of Szpilrajn type have been successfully applied to many branches. In economics, they play an important role in: $(i)$ revealed preference theory (cf., Richter [41]), (ii) the theory of choice (e.g., Chambers and Yenmez [16] use the Szpilrajn theorem to characterize responsive choice functions, that have been extensively used in matching theory and real matching markets) and social choice (e.g., in the analysis of social decision rules, the characterization of ordinal relations by Sholomov [43]; in the analysis of voting rules, Laruelle and Valenciano [35, Section 5] benefit from the Dushnik-Miller approach), see also Lahiri [34], Nehring and Puppe [38], Suzumura [44,45], Weymark [48] as a sample; (iii) aggregation of infinite utility streams, a field where one of its milestones, namely, Svensson's theorem [46], is a direct application of the Szpilrajn theorem; also in this field, Mabrouk [36, Section 4] obtains yet another consequence from his aforementioned result; or (iv) welfare economics, where Donaldson and Weymark [17] applied their extension theorem in a variety of examples. Further applications include game theory (cf., e.g., Bade [6]), computability theory (e.g., Roy [42] states the recursive version of the Szpilrajn theorem), order theory (Downey [18]), .... We address the reader to Bosi and Herden [9] for a more extensive list of applications.

In this fruitful field of research we can also name various fuzzy versions or extensions of Szpilrajn's theorem. Among them, Georgescu [25, Theorem 5.4], [26, Corollary 4.37] and [27, Theorem 4.17], Bodenhofer and Klawonn [7, Theorem 6.7] -who conduct a detailed investigation of linearity axioms for fuzzy orders-, Gottwald [28, Proposition 2.34], Höhle and Blanchard [31] -who pro-

\footnotetext{
${ }^{3}$ The case of finite sets is different. In such case, extensions à la Szpilrajn can be obtained in a somewhat constructive way, using classical techniques like "labelling": see, e.g., Caspard et al. [15].
} 
duce variations for antisymmetric preorders both of the extension theorem in their Theorem II.7 and of the intersection theorem in their Corollary II.8-, or Zadeh [51, Theorem 8].

In this paper we contribute to the field by providing several new variations of the Szpilrajn theorem for fuzzy preorders. We proceed in the spirit of Alcantud [4], thus we are concerned with the identification of constraints that can be imposed to the fuzzy order extensions of fuzzy relations. For the purpose of defining the key concept of compatible extension of a fuzzy reflexive relation, we consider the successful approach to the construction of an asymmetric part from the original relation that is based on generators (cf., De Baets and Fodor [12]). Firstly we give general extending results that reproduce useful statements in Georgescu [25] under the alternative construction here adopted. As an application, the recourse to 1-Lipschitz generators allows us to identify precisely which lists of pairs of connections with degree 1 by the asymmetric part can be imposed to the compatible extensions of a fuzzy preorder. The general case of imposing pairs of connections with degree 1 by either the asymmetric or the symmetric part is investigated under the generator min. This restriction permits to prove a sufficient condition that is not necessary. We also check that the only generator for which our proposed condition is sufficient is the minimum.

This paper is organized as follows. In Section 2 we recall the results by Alcantud [4] that inspire our investigation. We prove slightly more general versions of his two main theorems, stated for a wider class of binary relations. In Section 3 we give some notation and preliminaries on fuzzy relations and their extensions. Section 4 solves the problems posed. We briefly recall the main facts about (indifference) generators. Then we prove our results on unrestricted extensions in Subsection 4.1, and we apply them to tackle the aforementioned conditional extension problems in Subsection 4.2. Theorem 4 gives a precise answer for a particular specification of the problem under fairly general conditions, and Theorem 5 and the discussion afterwards concern the general case. In Section 5 we conclude and pose questions for further research.

\section{Crisp conditional extensions}

Alcantud [4] contributes to an original approach to conditional extensions in the crisp setting. It raises and solves the question: what do we need to check in order for a finite list of comparisons to be realized by an extension of a given preorder? The solutions are stated in terms of consistency properties for certain auxiliary binary relations. In this section we prove that the characterizations in [4] apply to a more general class of binary relations. First we recall some basic notions in the crisp set context.

Definition 1. A binary relation $Q$ on a universe $X$ is reflexive if aQa for all $a \in X$. A weak preference is a reflexive relation.

Associated with any weak preference relation $R$ we can build its symmetric component, called indifference relation and denoted by $I_{R}$ or simply $I$ when there 
is no danger of confusion. It is defined as $a I_{R} b$ if and only if $a R b \wedge b R a$. We can also obtain an asymmetric component, called strict preference relation and denoted by $P_{R}$ or simply $P$ when there is no danger of confusion. It is defined as $a P b$ if and only if $a R b \wedge \neg b R a$.

Weak preference relations $R$ are usually understood as follows: if $a$ is considered at least as good as $b$, then $a R b$, otherwise $\neg a R b$.

Definition 2. Given a binary relation $Q$ on a universe $X$, it is

(a) transitive if for all $a, b, c \in X$

$$
(a Q b \wedge b Q c) \Rightarrow a Q c,
$$

(b) acyclic if for any $a_{1}, \ldots, a_{n} \in X$ it holds that

$$
\left(a_{1} Q a_{2} \wedge a_{2} Q a_{3} \wedge \cdots \wedge a_{n-1} Q a_{n}\right) \quad \Rightarrow \quad \neg a_{n} Q a_{1},
$$

(c) transitive-consistent if it is a weak preference relation $R$ with strict preference relation $P$ such that for any $a_{1}, \ldots, a_{n} \in X$ it holds that

$$
\left(a_{1} P a_{2} \wedge a_{2} R a_{3} \wedge \cdots \wedge a_{n-1} R a_{n}\right) \quad \Rightarrow \quad \neg a_{n} R a_{1},
$$

(d) total if $a Q b \vee b Q a$ for all $a \neq b \in X$,

(e) complete if $a Q b \vee b Q a$ for all $a, b \in X$,

(f) a preorder if it is transitive and reflexive,

(g) an order if it is a total preorder.

Definition 3. The transitive closure of a relation $Q$ is the smallest transitive relation $Q^{T}$ such that $Q \subseteq Q^{T}$.

It is easy to prove that the transitive closure of a relation $Q$ is the intersection of all the transitive relations that contain the relation $Q$.

Definition 4. If $Q \subseteq Q^{\prime}$, then $Q^{\prime}$ is an extension of $Q$. Given a weak preference relation $R_{1}$, a compatible extension of $R_{1}$ is another weak preference relation $R_{2}$ defined on the same universe, such that $R_{1} \subseteq R_{2}$ and $P_{R_{1}} \subseteq P_{R_{2}}$, where $P_{R_{i}}$ is the strict preference relation associated with $R_{i}$.

Alcantud's first theorem is stated with the help of the following auxiliary relation:

Definition 5. Let $R$ be a binary relation defined on a set of alternatives $X$. Let $X_{I}=\left\{a_{1}, \ldots, a_{n}, b_{1}, \ldots, b_{n}\right\}$ be an ordered set of possibly repeated elements of $X$. The relation $R^{A}$ on $\left\{a_{1}, \ldots, a_{n}\right\}$ associated with $X_{I}$ and $R$ is defined by $a_{i} R^{A} a_{j}=a_{i} R b_{j}$.

Theorem 1 (Alcantud [4]). Let $R$ be a preorder on a set $X$. Let $X_{I}=$ $\left\{a_{1}, \ldots, a_{n}, b_{1}, \ldots, b_{n}\right\}$ be an ordered list of possibly repeated elements of $X$. The following statements are equivalent: 
a) There is an order $\tilde{R}$ that is a compatible extension of $R$ such that $b_{i} \tilde{P} a_{i}$ for each $i=1, \ldots, n$, where $\tilde{P}$ denotes the asymmetric part of $\tilde{R}$.

b) $R^{A}$ associated with $X_{I}$ and $R$ is acyclic.

In order to recall Alcantud's second theorem we need to introduce new concepts.

Definition 6. Let $X_{I}=\left\{a_{1}, \ldots, a_{p}, b_{1}, \ldots, b_{p}\right\}$ be an ordered list of possibly repeated elements of $X$. For each $c \in X_{I}$, let

$$
\delta(c)= \begin{cases}b_{i} & \text { if } c=a_{i}, \\ a_{i} & \text { if } c=b_{i} .\end{cases}
$$

Let $X_{I}^{n}=\left\{a_{n+1}, \ldots, a_{p}, b_{n+1}, \ldots, b_{p}\right\}$. If $n=p$, then $X_{I}^{n}=\varnothing$.

Definition 7. The relation $R^{I}$ associated with $R$ and $X_{I}$ is defined as follows:

$$
a R^{I} b=a R \delta(b), \text { for all } a, b \in X_{I} .
$$

We say that $R^{I}$ is $\delta$-cyclic along $X_{I}^{n}$ if $c_{1} R^{I} c_{2}$ and $c_{2} R^{I} c_{3}$ and $\ldots c_{k} R^{I} c_{1}$ implies that

$$
\delta\left(c_{1}\right) R^{I} \delta\left(c_{k}\right) \quad \ldots \quad \delta\left(c_{2}\right) R^{I} \delta\left(c_{1}\right), \text { for all } c_{1}, \ldots, c_{k} \in X_{I}^{n} .
$$

Definition 8. The relation $R^{G}$ associated with $R, n \leq p$ and $X_{I}$ is defined by

$$
a_{i} R^{G} a_{j}=\left(a_{i} R b_{j} \vee \bigvee_{\left\{c_{1}, \ldots c_{k}\right\} \subseteq X_{I}^{n}}\left(a_{i} R \delta\left(c_{1}\right) \wedge c_{1} R \delta\left(c_{2}\right) \wedge \cdots \wedge c_{k} R b_{j}\right)\right) .
$$

Definition 9. The relation $R^{G}$ is $\delta$-consistent with $X_{I}$ and $n \leq p$ if

$$
a_{i_{1}} R^{G} a_{i_{2}}, \ldots, a_{i_{k}} R^{G} a_{i_{1}} \text { implies } i_{t}>n \text { for all } t \in\{1, \ldots, k\} .
$$

Theorem 2 (Alcantud [4]). Let $R$ be a preorder on a set $X$. Let $X_{I}=$ $\left\{a_{1}, \ldots, a_{p}, b_{1}, \ldots, b_{p}\right\}$ be an ordered list of possibly repeated elements of $X$ and let $n \leq p$. The following statements are equivalent:

a) There exists an order $\tilde{R}$ that is a compatible extension of $R$ such that $b_{i} \tilde{P} a_{i}$ for each $i=1, \ldots, n$, and $b_{i} \tilde{I} a_{i}$ for $i=n+1, \ldots, p$, where $\tilde{P}$ and $\tilde{I}$ denote the asymmetric and symmetric components of $\tilde{R}$ respectively.

b) The relation $R^{G}$ is $\delta$-consistent with $X_{I}$ and $n$, and $R^{I}$ is $\delta$-cyclic along $X_{I}^{n}$.

Lemma 1. For every transitive-consistent weak preference relation $R$, its transitive closure $R^{T}$ is a compatible extension.

Proof. It is clear that $R \subseteq R^{T}$. We only have to prove that $P_{R} \subseteq P_{R^{T}}$. Consider a pair of alternatives such that $a P b$. Equivalently, $a R b$ and $\neg b R a$. The fact $a R b$ implies $a R^{T} b$. Assume by absurdum that $\neg a P_{R^{T}} b$, since $a R^{T} b$ then $b R^{T} a$. By definition of transitive closure, $b R^{T} a$ if and only if there exist $c_{1}, \ldots, c_{n} \in X$ such that $b R c_{1} \wedge c_{1} R c_{2} \wedge \cdots \wedge c_{n} R a$. But also $a P b$, which leads to

$$
a P b \wedge b R c_{1} \wedge c_{1} R c_{2} \wedge \cdots \wedge c_{n} R a .
$$

This is a contradiction, since $R$ is transitive-consistent. Our claim $a P_{R^{T}} b$ follows. 
Two more lemmas help to present Corollaries 1 and 2 below, the key results in this section.

Lemma 2. Let $R$ be a transitive-consistent weak preference relation on a universe $X$ and $R^{T}$ be its transitive closure. For any relation $\tilde{R}$ that is both transitive and a compatible extension of $R$, the following implications hold:

a) If $a R^{T} b$, then $a \tilde{R} b$.

b) If $a \tilde{I} b$, then $\neg a P^{T} b$ (and obviously $\neg b P^{T} a$ ),

where to alleviate the notation, $\tilde{P}$ and $\tilde{I}$ denote the asymmetric and symmetric components of $\tilde{R}$ respectively and $P^{T}$ and $I^{T}$ the asymmetric and symmetric components of $R^{T}$ respectively.

Proof. a) Since $R \subseteq \tilde{R}$ and $\tilde{R}$ is transitive, the implication follows from the fact that $R^{T}$ is the transitive closure of $R$, because the transitive closure is the intersection of all the transitive relations that extend $R$.

$b$ ) By definition, $a \tilde{I} b$ is equivalent to $a \tilde{R} b$ and $b \tilde{R} a$. Assume that $a P^{T} b$. We will get a contradiction. $a P^{T} b$ is equivalent to $a R^{T} b \wedge \neg b R^{T} a$.

Since $R^{T}$ is a compatible extension of $R$, it cannot happen that $b P a$, otherwise also $b \tilde{P} a$, but this contradicts the assumption $a \tilde{P} b$. So $\neg b P a$. The same happens if we assume $a I b$, then also $a \tilde{I} b$ and this contradicts $a \tilde{P} b$. Thus $\neg a R b$. Since $a R^{T} b$, there exist $c_{1}, \ldots, c_{n}$ elements in $X$ such that

$$
a R c_{1} \wedge c_{1} R c_{2} \wedge \quad \ldots \wedge c_{n} R b
$$

This implies

$$
a \tilde{R} c_{1} \wedge c_{1} \tilde{R} c_{2} \wedge \quad \ldots \wedge c_{n} \tilde{R} b
$$

which jointly with $b \tilde{R} a$ and the fact that $\tilde{R}$ is transitive leads to $c_{i} \tilde{I} c_{i+1}$ for all $i=0, \ldots, n$ where $c_{0}=a$ and $c_{n+1}=b$. It suffices to prove that $c_{i+1} \tilde{R} c_{i}$ for all $i$. But this follows from

$$
c_{i+1} \tilde{R} c_{i+2} \wedge \cdots \wedge c_{n} \tilde{R} b \wedge b \tilde{R} a \wedge a \tilde{R} c_{1} \wedge \cdots \wedge c_{i-1} \tilde{R} c_{i}
$$

and the transitivity of $\tilde{R}$. Since $c_{i} \tilde{R} c_{i+1}$ and $c_{i+1} \tilde{R} c_{i}$, then $c_{i} \tilde{I} c_{i+1}$ for all $i=0, \ldots, n$. This implies $\neg c_{i} \tilde{P} c_{i+1}$ for any $i$. Since $P \subseteq \tilde{P}$, one has $\neg c_{i} P c_{i+1}$ for all $i$, but according to Eq. (1) $c_{i} R c_{i+1}$ for all $i$. This means $c_{i} I c_{i+1}$ for all $i=0, \ldots, n$, or equivalently, $c_{i} R c_{i+1}$ and $c_{i+1} R c_{i}$. This implies $c_{i} R^{T} c_{i+1}$ and $c_{i+1} R^{T} c_{i}$ for all $i$. Since $R^{T}$ is transitive, we can deduce $a R^{T} b$ and $b R^{T} a$. Equivalently, $a I^{T} b$. A contradiction, since we started assuming $b P^{T} a$.

Lemma 3. Let $R$ be a transitive-consistent weak preference relation on a universe $X$ and $R^{T}$ its transitive closure. A transitive relation $\tilde{R}$ is a compatible extension of $R$ if and only if it is a compatible extension of $R^{T}$.

Proof. The "If" part is a direct consequence of Lemma 1. To prove the "Only if" part , assume that $\tilde{R}$ is a transitive relation that is a compatible extension of $R$. We keep the notation of Lemma 2, thus $\tilde{P}$ and $P^{T}$ denote the asymmetric 
components of $\tilde{R}$ and $R^{T}$ respectively. Lemma $2 a$ ) proves $R^{T} \subseteq \tilde{R}$ thus we only need to prove $P^{T} \subseteq \tilde{P}$. Assume $a P^{T} b$, then $a R^{T} b$ and also $a \tilde{R} b$ because $R^{T} \subseteq \tilde{R}$. According to Lemma $\left.2 b\right), a P^{T} b$ implies $\neg a \tilde{I} b$, which coupled with $a \tilde{R} b$ implies $a \tilde{P} b$.

We are ready to slightly extend Theorems 1 and 2 . We prove that they not only apply to preorders, but also to reflexive and transitive-consistent binary relations.

Corollary 1. Let $R$ be a (crisp) transitive-consistent weak preference relation on a universe $X$. Let $X_{I}=\left\{a_{1}, \ldots, a_{n}, b_{1}, \ldots, b_{n}\right\}$ be an ordered list of possibly repeated elements of $X$. The following statements are equivalent:

a) There is an order $\tilde{R}$ that is a compatible extension of $R$ such that $b_{i} \tilde{P} a_{i}$ for each $i=1, \ldots, n$, where $\tilde{P}$ denotes the asymmetric component of $\tilde{R}$.

b) $R^{A}$ associated with $X_{I}$ and $R^{T}$ is acyclic.

Proof. The equivalence follows from Lemma 3 and Theorem 1.

Corollary 2. Let $R$ be a (crisp) transitive-consistent weak preference relation on a universe $X$. Let $X_{I}=\left\{a_{1}, \ldots, a_{p}, b_{1}, \ldots, b_{p}\right\}$ be an ordered list of possibly repeated elements of $X$ and let $n \leq p$. The following statements are equivalent:

a) There exists an order $\tilde{R}$ that is a compatible extension of $R$ such that $b_{i} \tilde{P} a_{i}$ for each $i=1, \ldots, n$ and $b_{i} \tilde{I} a_{i}$ for $i=n+1, \ldots, p$.

b) The relation $R^{G}$ associated with $R^{T}$ is $\delta$-consistent with $X_{I}$ and $n$, and $R^{I}$ (associated with $R^{T}$ ) is $\delta$-cyclic along $X_{I}^{n}$.

Proof. The equivalence follows from Lemma 3 and Theorem 2.

\section{$3 \quad$ Fuzzy relations and their extensions}

In this section we introduce basic definitions on fuzzy set theory.

Fuzzy set theory was introduced by Zadeh in [50]. The aim was to formalize the human way of thinking and acting. Up to then mathematics and logic were crisp: only true/false assertions could be written formally. However, our way of thinking is much more ambiguous, there is not only black and white. Specially feelings are usually expressed with phrases as "I like it a bit", "slightly prefer" or "do not like it too much". Fuzzy sets and in particular fuzzy relations were introduced to grasp those nuances that classical mathematics cannot express. Fuzzy relations can take any value in the $[0,1]$ interval. The value shows the strength of the connection between the alternatives.

Definition 10. A fuzzy relation $Q$ defined on a universe $X$ is a mapping $Q$ : $X \times X \rightarrow[0,1]$ such that for every $a, b \in X, Q(a, b)$ indicates the degree with which $a$ is connected to $b$ by the relation $Q$.

A fuzzy weak preference relation is a reflexive fuzzy relation, i.e., a fuzzy relation $R$ satisfying $R(a, a)=1$ for all $a \in X$. 
The classical connectives must also be generalized in order to deal with fuzzy relations. Intersection and union of fuzzy sets are usually based on t-norms and t-conorms. A t-norm $T$ is a binary mapping $T:[0,1] \times[0,1] \rightarrow[0,1]$ satisfying the following four properties: commutativity, associativity, monotonicity (in each component) and neutral element 1 . The greatest t-norm is the minimum operator $T_{\mathbf{M}}(x, y)=\min (x, y)$. We also use the common notation $\min (x, y)=x \wedge y$. Another two important t-norms are the Eukasiewicz operator defined as $T_{\mathbf{L}}(x, y)=$ $\max \{x+y-1,0\}$ and the product, defined as $T_{\mathbf{P}}(x, y)=x \cdot y$.

A t-norm $T$ has zero divisors if there is a pair of values $(x, y) \in] 0,1\left[{ }^{2} \mathrm{such}\right.$ that $T(x, y)=0$. In this case $x$ and $y$ are called zero divisors of $T$. The minimum and product t-norms do not admit zero divisors. For the Eukasiewicz t-norm every pair of values $(x, y)$ such that $x+y \leq 1$ are zero divisors. For the drastic t-norm every pair of values $(x, y) \in] 0,1\left[{ }^{2}\right.$ are zero divisors.

The intersection of fuzzy relations is defined using t-norms: given a t-norm $T$, the $T$-intersection of the fuzzy relations $Q_{1}$ and $Q_{2}$ on the universe $X$ is denoted by $Q_{1} \cap_{T} Q_{2}$, defined by $\left(Q_{1} \cap_{T} Q_{2}\right)(a, b)=T\left(Q_{1}(a, b), Q_{2}(a, b)\right)$ for all $a, b \in X$.

A t-conorm $S$ is a mapping $S:[0,1] \times[0,1] \rightarrow[0,1]$ satisfying similar properties to t-norms: commutativity, associativity, monotonicity (in each component) and neutral element 0 . The smallest t-conorm is the maximum operator $S_{\mathbf{M}}(x, y)=\max (x, y)$. As for the minimum, the alternative notation $x \vee y$ is commonly used to express the maximum between $x$ and $y$.

The Eukasiewicz t-conorm is $S_{\mathbf{L}}(x, y)=\min \{x+y, 1\}$ and the product $\mathrm{t}$ conorm is $S_{\mathbf{P}}(x, y)=x+y-x y$.

The union of fuzzy relations is defined using t-conorms: given a t-conorm $S$, the $S$-union of the fuzzy relations $Q_{1}$ and $Q_{2}$ on the universe $X$ is denoted by $Q_{1} \cup_{S} Q_{2}$, defined by $\left(Q_{1} \cup_{S} Q_{2}\right)(a, b)=S\left(Q_{1}(a, b), Q_{2}(a, b)\right)$ for all $a, b \in X$.

For a complete study on t-norms and t-conorms see [33].

The standard negation of a fuzzy relation is $\neg R$ defined as $\neg R(a, b)=1-$ $R(a, b)$.

Let $T$ be a t-norm. The $T$-composition of two fuzzy relations $Q_{1}$ and $Q_{2}$ is denoted by $Q_{1} \circ_{T} Q_{2}$, and it is defined by $\left(Q_{1} \circ_{T} Q_{2}\right)(a, b)=\sup _{c \in X} T\left(Q_{1}(a, c), Q_{2}(c, b)\right)$ for all $a, b \in X$.

A fuzzy relation $Q$ is $T$-transitive if

$$
T(Q(a, b), Q(b, c)) \leq Q(a, c), \quad \text { for all } a, b, c \in X
$$

Thus there are several alternative definitions of transitivity for fuzzy relations which in general are not equivalent, since the concept is conditional on the tnorm. Montes et al. [37] is a good example of the relevance of the t-norm chosen. The $T$-transitivity of a fuzzy relation $Q$ is usually denoted by $Q \circ_{T} Q \subseteq Q$. In particular, min-transitivity means $\min (Q(a, b), Q(b, c)) \leq Q(a, c)$ for all $a, b, c \in$ $X$.

The min-transitive closure of a fuzzy relation $Q$ is the smallest min-transitive fuzzy relation that contains $Q$. We denote it by $Q^{T}$, the capital $T$ standing for transitivity. It is well known that $Q^{T}=\bigcap\left\{Q_{i} \supseteq Q \mid Q_{i}\right.$ min-transitive $\}$. 
The notion of completeness admits different generalizations too. They are usually based on t-conorms. In this contribution we consider the weakest type of completeness: we say that a fuzzy relation $R$ is total if for all $a \neq b \in X$ it holds that $R(a, b) \vee R(b, a)>0$. Observe that a reflexive relation $R$ is total if and only if $R(a, b) \vee R(b, a)>0$ for all $a, b \in X$.

Definition 11. We say that a fuzzy relation $R$ is a fuzzy preorder if it is reflexive and min-transitive. A fuzzy order $R$ is a fuzzy total preorder.

Definition 12. A fuzzy weak preference relation $R$ with an associated strict preference relation $P$ is T-transitive-consistent if

$T\left(P\left(a_{1}, a_{2}\right), R\left(a_{2}, a_{3}\right), \ldots, R\left(a_{n-1}, a_{n}\right)\right) \leq \neg R\left(a_{n}, a_{1}\right) \quad$ for all $a_{1}, \ldots, a_{n} \in X$.

For the minimum t-norm, one has that $R$ is min-transitive-consistent if and only if for every $a_{1}, \ldots, a_{n} \in X$,

$$
\min \left(P\left(a_{1}, a_{2}\right), R\left(a_{2}, a_{3}\right), \ldots, R\left(a_{n-1}, a_{n}\right)\right) \leq 1-R\left(a_{n}, a_{1}\right) .
$$

Later on in this paper we discuss the connection between transitive-consistency and transitivity in the fuzzy context.

Definition 13. A fuzzy relation $Q$ is T-acyclic if

$$
T\left(Q\left(a_{1}, a_{2}\right), Q\left(a_{2}, a_{3}\right), \ldots, Q\left(a_{n}, a_{1}\right)\right)=0 \quad \text { for all } a_{1}, \ldots, a_{n} \in X .
$$

Definition 14. A fuzzy relation $\tilde{R}$ is a compatible extension of the fuzzy weak preference relation $R$ on $X$ if and only if $R(a, b) \leq \tilde{R}(a, b)$ and $P(a, b) \leq \tilde{P}(a, b)$ for all $a, b \in X$.

Unless otherwise stated, the results proved in the sequel refer to min-transitivity, i.e., the transitivity based on the minimum t-norm. Consequently, for a fuzzy relation $Q$, min-transitivity amounts to $Q \circ Q \subseteq Q$.

\section{Fuzzy extensions with constraints: a study based on generators}

Our study is motivated by Remark 1 below, which establishes that constraints cannot be imposed on the extensions with impunity.

Remark 1. Consider the fuzzy preorder $R$ defined on $X=\{a, b, c, d\}$ through $R(a, b)=1, R(c, d)=1, R(x, x)=1$ for all $x \in X$, and $R(x, y)=0$ otherwise. Then there exists no fuzzy order $\tilde{R}$ that is an extension of $R$ and satisfies both $\tilde{R}(a, d)=0$ and $\tilde{R}(c, b)=0$. To prove it, observe that because $\tilde{R}(a, d)=0$ and $\tilde{R}$ should be total, then $\tilde{R}(d, a)>0$. By transitivity, $\tilde{R}(d, b) \geq$ $\min (\tilde{R}(d, a), \tilde{R}(a, b))>0$ thus $\tilde{R}(c, b) \geq \min (\tilde{R}(c, d), \tilde{R}(d, b))>0$, this is a contradiction. 
In order to cope with the problem that arises when constraints must be accounted for, in this section we expand the proposal by Alcantud [4] (v. Section 2) to the analysis of fuzzy relations.

Preliminarily, in Subsection 4.1 we prove some useful results in the context of the general extension problem, that is to say, of the direct fuzzy analogue of Szpilrajn's extension problem (in Hansson's form). The main contribution is Theorem 3 below, which is subsequently used in Subsection 4.2. Here Theorems 4 and 5 benefit from this tool in order to generalize the approach to conditional extensions for crisp relations presented in Section 2 .

Before formulating the statements, the notion of compatible extension must be specified. This in turn relies on the concept of an asymmetric component of a fuzzy relation. Unlike the crisp case, in the fuzzy context there is not a universally accepted procedure to derive asymmetric and symmetric components of a relation. This has been a topic of debate for many years (see e.g., [13, 21, 39, $40])$. Fodor and Roubens proposed an axiomatic construction [22, 23] and some years later De Baets and Fodor [12] introduced the most successful proposal based on (indifference) generators, which we proceed to recall.

Definition 15. A generator $i$ is a commutative binary operation on the unit interval $[0,1]$ that is bounded by the Lukasiewicz t-norm $T_{\mathbf{L}}$ and the minimum operator $T_{\mathbf{M}}$, i.e. $T_{\mathbf{L}} \leq i \leq T_{\mathbf{M}}$.

Since generators are not necessarily associative, they are not necessarily tnorms.

Recall that a binary operation $f:[0,1]^{2} \rightarrow[0,1]$ satisfies the 1-Lipschitz property if for each $\left(x_{1}, y_{1}\right),\left(x_{2}, y_{2}\right) \in[0,1]^{2}$,

$$
\left|f\left(x_{1}, y_{1}\right)-f\left(x_{2}, y_{2}\right)\right| \leq\left|x_{1}-x_{2}\right|+\left|y_{1}-y_{2}\right|
$$

As a consequence, if $x_{1} \leq x_{2}$, then $x_{2}-x_{1} \geq f\left(x_{2}, y\right)-f\left(x_{1}, y\right)$ for any $y \in[0,1]$. Equivalently, for any $x_{1} \leq x_{2}$ and $y \in[0,1]$

$$
x_{2}-f\left(x_{2}, y\right) \geq x_{1}-f\left(x_{1}, y\right) \text {. }
$$

The minimum operator (among others) satisfies this property, which is slightly stronger than continuity. The 1-Lipschitz property plays a key role in the remaining of this section.

Fix a generator $i$. For any reflexive fuzzy relation $R$ on $X$ we can define the indifference and strict preference relations associated with $R$ as follows:

$$
I(a, b)=i(R(a, b), R(b, a)), \quad P(a, b)=R(a, b)-i(R(a, b), R(b, a))
$$

Irrespective of the generator, the relation $R$ can be expressed as the union (by the Eukasiewicz t-conorm) of $P$ and $I$, as in the classical case: $R=P+I{ }^{4}$

We proceed to prove by examples that min-transitive-consistency is logically unrelated to min-transitivity when generators are involved:

\footnotetext{
${ }^{4}$ From a formal point of view, because we get a different pair $(P, I)$ for each generator it seems necessary to express dependence on the generator. However we believe that no confusion arises by avoiding cumbersome notation.
} 
Example 1. Consider the fuzzy min-transitive relation $R$ defined on $\{a, b, c\}$ as follows. Fix now any generator, the associated strict preference relation $P$ is shown on the right:

\begin{tabular}{|c|c|c|c|c|}
\hline$R$ & $\begin{array}{lll}a & b & c\end{array}$ & $P$ & $a$ & $b \quad c$ \\
\hline$a$ & $\begin{array}{lll}1 & 0.21\end{array}$ & $a$ & 0 & 00.8 \\
\hline$b$ & $\begin{array}{lll}1 & 1 & 1\end{array}$ & $b$ & 0.8 & $\begin{array}{ll}0 & 0.8\end{array}$ \\
\hline$c$ & 0.20 .21 & $c$ & 0 & 0 \\
\hline
\end{tabular}

It holds that $R$ is min-transitive, but it is not min-transitive-consistent,

$$
\min (P(a, c), R(c, b))=\min (0.8,0.2)=0.2 \not \leq 0=\neg R(b, a) .
$$

Now consider the weak preference relation $R$ and its associated strict preference relation $P$ (by means of any generator):

\begin{tabular}{|c|c|c|c|c|c|}
\hline$R$ & $\begin{array}{ll}a & b\end{array}$ & $c$ & $P$ & $\begin{array}{ll}a & b\end{array}$ & $c$ \\
\hline$a$ & 10.8 & 0.1 & $a$ & $\begin{array}{ll}0 & 0.8\end{array}$ & 0.1 \\
\hline$b$ & $\begin{array}{ll}0 & 1\end{array}$ & 0.8 & $b$ & $\begin{array}{ll}0 & 0\end{array}$ & 0.8 \\
\hline$c$ & $\begin{array}{ll}0 & 0\end{array}$ & 1 & $c$ & 0 & 0 \\
\hline
\end{tabular}

It is easy to check that $R$ is min-transitive-consistent. However, it is not mintransitive because $R(a, c)=0.1 \nsupseteq \min (0.8,0.8)=\min (R(a, b), R(b, c))$.

The aforementioned logical independence of transitive-consistency and transitivity prevents us from obtaining a fuzzy version of Corollaries 1 and 2. However, we still can obtain a fuzzy extension of Theorems 1 and 2 in [4] by appealing to further preliminary results that we proceed to discuss in Subsection 4.1.

\subsection{A general result on compatible extensions}

The aim of this subsection is to prove a general result on compatible extensions, the concept depending on a 1-Lipschitz generator.

Let us introduce some definitions. The relation $R[a, b]$ associated with $R$ and $a, b \in X$ is defined as

$$
R[a, b](c, d)=R(c, d) \text { if }(c, d) \neq(a, b), \quad R[a, b](c, d)=1 \text { if }(c, d)=(a, b) .
$$

The relation $R[a, b]^{T}$ is the transitive closure of $R[a, b]$.

Proposition 1. Let us fix a 1-Lipschitz generator $i$, and assume that all indifference and strict preference relations are derived by Eq. (3) via $i$.

If $R$ is a fuzzy preorder such that $R(b, a)=0$ then $R[a, b]^{T}$ is a compatible extension of $R$.

Proof. It is clear that $R \subseteq R[a, b]^{T}$. Let us prove $P \subseteq P[a, b]^{T}$, where $P[a, b]^{T}$ denotes the asymmetric component of $R[a, b]^{T}$.

Take $(c, d)$ such that $P(c, d)>0$. Then $R[a, b](c, d) \geq R(c, d)>0$. 
If $R[a, b]^{T}(d, c)=R(d, c)$ then

$$
\begin{aligned}
P[a, b]^{T}(c, d) & =R[a, b]^{T}(c, d)-i\left(R[a, b]^{T}(c, d), R[a, b]^{T}(d, c)\right) \\
& =R[a, b]^{T}(c, d)-i\left(R[a, b]^{T}(c, d), R(d, c)\right) \\
& \geq R(c, d)-i(R(c, d), R(d, c))=P(c, d)
\end{aligned}
$$

where the desired inequality follows from Eq. (2).

Otherwise $R[a, b]^{T}(d, c)>R(d, c)$. We prove that this leads to a contradiction by distinguishing two cases.

Case 1: If $R[a, b](d, c)>R(d, c)$ then a fortiori $(d, c)=(a, b)$. But in this case $R(c, d)=R(b, a)$, against the fact that $R(b, a)=0$ and $R(c, d)>0$.

Case 2: If $R[a, b]^{T}(d, c)>R[a, b](d, c)=R(d, c)$ then there must exist $c_{1}, \ldots, c_{n}$ such that

$$
R[a, b](d, c)<\min \left(R[a, b]\left(d, c_{1}\right), \ldots, R[a, b]\left(c_{n}, c\right)\right)
$$

If $\left(c_{i}, c_{i+1}\right) \neq(a, b)$ for all $i$, then $R[a, b]\left(c_{i}, c_{i+1}\right)=R\left(c_{i}, c_{i+1}\right)$ and by the transitivity of $R$ we obtain the contradiction

$$
\begin{aligned}
R[a, b](d, c) & \geq R(d, c) \geq \min \left(R\left(d, c_{1}\right), \ldots, R\left(c_{n}, c\right)\right) \\
& =\min \left(R[a, b]\left(d, c_{1}\right), \ldots, R[a, b]\left(c_{n}, c\right)\right) .
\end{aligned}
$$

Assume now that $\left(c_{i}, c_{i+1}\right)=(a, b)$ for some $i$. In this case

$$
\begin{aligned}
R(b, a) \geq & \min \left(R\left(b, c_{i+2}\right), \ldots, R\left(c_{n}, c\right), R(c, d), R\left(d, c_{1}\right), \ldots, R\left(c_{i-1}, a\right)\right) \\
= & \min \left(R[a, b]\left(b, c_{i+2}\right), \ldots, R[b, a]\left(c_{n}, c\right), R(c, d),\right. \\
& \left.R[a, b]\left(d, c_{1}\right), \ldots, R[a, b]\left(c_{i-1}, a\right)\right)>0 .
\end{aligned}
$$

This is a contradiction since $R(b, a)=0$. This completes the proof.

Before proving our main result in this subsection, we need an auxiliary lemma which uses the following concept: a set of fuzzy relations $\mathcal{R}$ is closed upward if for any totally ordered family (by set inclusion) $\left\{R_{i}\right\}_{i \in I}$ of fuzzy relations in $\mathcal{R}$, we have $\cup_{i \in I} R_{i} \in \mathcal{R}$.

Lemma 4 (Georgescu [25]). The set of transitive relations defined on a set $X$ is closed upward. The intersection of two closed upward sets is closed upward. For any fuzzy weak preference relation $R$, the set of compatible extensions of $R$ is closed upward.

Theorem 3. Let us fix a 1-Lipschitz generator $i$, and assume that all indifference and strict preference relations are derived by Eq. (3) via $i$.

For any fuzzy preorder $R$ defined on a universe $X$, there exists a total and transitive fuzzy relation $\tilde{R}$ such that $\tilde{R}$ is a compatible extension of $R$.

Proof. The set of transitive relations that are a compatible extension of $R$ is the intersection of the set of transitive relations in $X$ and the set of compatible extensions of $R$ in $X$. Therefore by Lemma 4, we can assure that it is closed 
upward. Zorn's Lemma ensures the existence of a maximal element $\tilde{R}$ in that set, i.e., $\tilde{R}$ is maximal (by set inclusion) in the set of transitive relations that are a compatible extension of $R$. We only need to prove that $\tilde{R}$ is total.

By way of contradiction, assume $\tilde{R}(a, b)=\tilde{R}(b, a)=0$ for some $a, b \in X$. By Proposition $1, \tilde{R}[a, b]^{T}$ is both transitive and a compatible extension of $\tilde{R}$, therefore of $R$. Because $\tilde{R}[a, b]^{T}(a, b)=1>0=\tilde{R}(a, b)$, the contradiction $\tilde{R} \subsetneq \tilde{R}[a, b]^{T}$ follows.

Remark 2. Proposition 1 and Theorem 3 emulate statements proven by Georgescu in $[25,27]$, the key difference being that here we deal with strict preference relations obtained from weak preference relations by 1-Lipschitz generators, which is different from her construction.

\subsection{Compatible extensions with constraints}

We are now ready to prove a fuzzy counterpart of the extension of Theorem 1 proved in Corollary 1, namely Theorem 4 below, which leans on 1-Lipschitz generators and the following definition inspired by Definition 5 :

Definition 16. Let $R$ be a fuzzy relation defined on a set of alternatives $X$. Let $X_{I}=\left\{a_{1}, \ldots, a_{n}, b_{1}, \ldots, b_{n}\right\}$ be an ordered set of possibly repeated elements of $X$. The fuzzy relation $R^{A}$ associated with $X_{I}$ and $R$ is defined by $R^{A}\left(a_{i}, a_{j}\right)=$ $R\left(a_{i}, b_{j}\right)$.

Theorem 4. Let us fix a 1-Lipschitz generator $i$, and assume that all indifference and strict preference relations are derived by Eq. (3) via $i$.

Let $\left\{a_{1}, \ldots, a_{n}, b_{1}, \ldots, b_{n}\right\}$ be a subset of possibly repeated elements of $X$ and let $R$ be a fuzzy preorder on $X$. The following statements are equivalent:

a) There exists an order $\tilde{R}$ that is a compatible extension of $R$ and such that $\tilde{P}\left(b_{i}, a_{i}\right)=1$ for all $i=1, \ldots, n$, where $\tilde{P}$ is the asymmetric relation obtained from $\tilde{R}$.

b) The relation $R^{A}$ associated with $\left\{a_{1}, \ldots, a_{n}, b_{1}, \ldots, b_{n}\right\}$ and $R$ is min-acyclic.

Proof. Let us assume that $a$ ) is true but $b$ ) does not hold. Then there exists a list of alternatives $a_{i_{1}}, \ldots, a_{i_{k}}$ such that

$$
R^{A}\left(a_{i_{1}}, a_{i_{2}}\right)>0, R^{A}\left(a_{i_{2}}, a_{i_{3}}\right)>0, \ldots, R^{A}\left(a_{i_{k}}, a_{i_{1}}\right)>0
$$

or equivalently,

$$
R\left(a_{i_{1}}, b_{i_{2}}\right)>0, R\left(a_{i_{2}}, b_{i_{3}}\right)>0, \ldots, R\left(a_{i_{k}}, b_{i_{1}}\right)>0 .
$$

In particular the latter inequalities yield

$$
\tilde{R}\left(a_{i_{1}}, b_{i_{2}}\right)>0, \tilde{R}\left(a_{i_{2}}, b_{i_{3}}\right)>0, \ldots, \tilde{R}\left(a_{i_{k}}, b_{i_{1}}\right)>0 .
$$

On the other hand, $\tilde{P}\left(b_{i_{j}}, a_{i_{j}}\right)=1$ for all $j=1, \ldots, k$ by our assumption. Therefore because the inequalities

$$
\tilde{R}\left(a_{i_{1}}, b_{i_{2}}\right)>0, \tilde{P}\left(b_{i_{2}}, a_{i_{2}}\right)>0, \ldots, \tilde{R}\left(a_{i_{k}}, b_{i_{1}}\right)>0
$$


have been established we obtain by min-transitivity that $\tilde{R}\left(a_{i_{1}}, b_{i_{1}}\right)>0$. This contradicts the fact that $\tilde{P}\left(b_{i_{1}}, a_{i_{1}}\right)=1$.

Now let us assume that $b$ ) is true. In order to prove $a$ ) we proceed in several steps.

Define the relation $\bar{R}$ as follows: for each $a, b \in X$,

$$
\bar{R}(a, b)= \begin{cases}R(a, b) & \text { if }(a, b) \neq\left(b_{i}, a_{i}\right) \text { for all } i \\ 1 & \text { otherwise }\end{cases}
$$

Then we prove the following claim:

Claim. For each $a, b \in X$, if there exist $c_{1} \ldots, c_{k}$ alternatives in $X$ such that

$$
\min \left(\bar{R}\left(a, c_{1}\right), \ldots, \bar{R}\left(c_{k}, b\right)\right)>0
$$

then either

$$
R(a, b) \geq \min \left(\bar{R}\left(a, c_{1}\right), \ldots, \bar{R}\left(c_{k}, b\right)\right)
$$

or

$$
\exists\left\{j_{1}, \ldots, j_{l}\right\} \subseteq\{1, \ldots, n\} \mid \min \left(R\left(a, b_{j_{1}}\right), R\left(a_{j_{1}}, b_{j_{2}}\right), \ldots, R\left(a_{j_{l}}, b\right)\right)>0 .
$$

To prove the claim, let $c_{0}=a$ and $c_{k+1}=b$. We distinguish two cases.

If $\left(c_{i}, c_{i+1}\right) \neq\left(b_{j}, a_{j}\right)$ for any $j \in\{1, \ldots, n\}$, then $\bar{R}\left(c_{i}, c_{i+1}\right)=R\left(c_{i}, c_{i+1}\right)$ for all $i$ and since $R$ is min-transitive, $R(a, b) \geq \min \left(R\left(a, c_{1}\right), \ldots, R\left(c_{k}, b\right)\right)=$ $\min \left(\bar{R}\left(a, c_{1}\right), \ldots, \bar{R}\left(c_{k}, b\right)\right)$.

This provides the statement in Eq. (4).

Otherwise there are $l$ indexes $\left\{i_{1}, \ldots, i_{l}\right\} \subseteq\{1, \ldots, n\}$ such that

$$
\begin{aligned}
& \left(c_{i_{1}}, c_{i_{1}+1}\right)=\left(b_{j_{1}}, a_{j_{1}}\right), \\
& \ldots, \\
& \left(c_{i_{l}}, c_{i_{l}+1}\right)=\left(b_{j_{l}}, a_{j_{l}}\right)
\end{aligned}
$$

and $\left(c_{i}, c_{i+1}\right) \neq\left(b_{j}, a_{j}\right)$ for all $i \notin\left\{i_{1}, \ldots, i_{l}\right\}$ and $j \in\{1, \ldots, n\}$. Therefore $\bar{R}\left(c_{i}, c_{i+1}\right)=R\left(c_{i}, c_{i+1}\right)$ for all $i \notin\left\{i_{1}, \ldots, i_{l}\right\}$. Since $R$ is min-transitive,

$$
\begin{aligned}
R\left(c_{i_{1}+1}, c_{i_{2}}\right) & \geq \min \left(R\left(c_{i_{1}+1}, c_{i_{1}+2}\right), \ldots, R\left(c_{i_{2}-1}, c_{i_{2}}\right)\right) \\
& =\min \left(\bar{R}\left(c_{i_{1}+1}, c_{i_{1}+2}\right), \ldots, \bar{R}\left(c_{i_{2}-1}, c_{i_{2}}\right)\right)>0 .
\end{aligned}
$$

A similar reasoning establishes $R\left(c_{i_{2}+1}, c_{i_{3}}\right)>0, \ldots, R\left(c_{i_{l-1}+1}, c_{i_{l}}\right)>0$. Furthermore, if $a \neq c_{i_{1}}$ then $R\left(a, c_{i_{1}}\right) \geq \min \left(R\left(a, c_{1}\right), \ldots, R\left(c_{i_{1}-1}, c_{i_{1}}\right)\right)>0$ and if $c_{i_{l}} \neq b$ then $R\left(c_{i_{l}+1}, b\right)>0$. Thus we get

$$
\min \left(R\left(a, c_{i_{1}}\right), R\left(c_{i_{1}+1}, c_{i_{2}}\right), \ldots, R\left(c_{i_{l}+1}, b\right)\right)>0
$$

which can be expressed as follows:

$$
\min \left(R\left(a, b_{j_{1}}\right), R\left(a_{j_{1}}, b_{j_{2}}\right), \ldots, R\left(a_{j_{l}}, b\right)\right)>0 .
$$

This provides the statement in Eq. (5). 
Consider now the transitive closure of $\bar{R}$, namely, the relation $\bar{R}^{T}$. We claim that this relation is a compatible extension of $R$. It is clear that $R \subseteq \bar{R}^{T}$ because $R \subseteq \bar{R} \subseteq \bar{R}^{T}$. Let us prove $P \subseteq \bar{P}^{T}$, where $\bar{P}^{T}$ stands for the asymmetric relation associated with $\bar{R}^{T}$ by the generator $i$. To that purpose, fix $(a, b)$ such that $P(a, b)>0$, thus $\bar{R}^{T}(a, b) \geq R(a, b)>0$. We distinguish two cases.

Case 1: $\bar{R}^{T}(b, a)=R(b, a)$. Then

$$
\begin{aligned}
\bar{P}^{T}(a, b) & =\bar{R}^{T}(a, b)-i\left(\bar{R}^{T}(a, b), \bar{R}^{T}(b, a)\right)=\bar{R}^{T}(a, b)-i\left(\bar{R}^{T}(a, b), R(b, a)\right) \\
& \geq R(a, b)-i(R(a, b), R(b, a))=P(a, b)
\end{aligned}
$$

where the desired inequality follows from the fact that $i$ is 1-Lipschitz and $\bar{R}^{T}(a, b) \geq R(a, b)$.

Case 2: $\bar{R}^{T}(b, a) \neq R(b, a)$, thus $\bar{R}^{T}(b, a)>R(b, a)$. Let us prove that this is absurd. It must be the case that $\bar{R}(b, a)=R(b, a)$ because otherwise $(b, a)=\left(b_{i}, a_{i}\right)$ for some $i$ and $R\left(a_{i}, b_{i}\right)=R(a, b)>0$ contradicts the fact that $R^{A}$ is min-acyclic (for $R^{A}\left(a_{i}, a_{i}\right)>0$ ). Therefore one has $\bar{R}^{T}(b, a)>\bar{R}(b, a)$. By the definition of transitive closure, there exist $c_{1}, \ldots, c_{k}$ alternatives such that

$$
\min \left(\bar{R}\left(b, c_{1}\right), \bar{R}\left(c_{1}, c_{2}\right), \ldots, \bar{R}\left(c_{k}, a\right)\right)>\bar{R}(b, a)>0 .
$$

By using the Claim above, the previous inequality leads to either

$$
R(b, a) \geq \min \left(\bar{R}\left(b, c_{1}\right), \ldots, \bar{R}\left(c_{k}, a\right)\right)
$$

or

$$
\exists\left\{i_{1}, \ldots, i_{l}\right\} \subseteq\{1, \ldots, n\} \mid \min \left(R\left(b, b_{i_{1}}\right), R\left(a_{i_{1}}, b_{i_{2}}\right), \ldots, R\left(a_{i_{l}}, a\right)\right)>0 .
$$

The first possibility gives rise to the contradiction

$$
R(b, a) \geq \min \left(\bar{R}\left(b, c_{1}\right), \bar{R}\left(c_{1}, c_{2}\right), \ldots, \bar{R}\left(c_{k}, a\right)\right)>\bar{R}(b, a) .
$$

Now assume the second possibility. Then

$$
\left.\min \left(R\left(a_{i_{1}}, b_{i_{2}}\right)\right), R\left(a_{i_{2}}, b_{i_{3}}\right), \ldots, R\left(a_{i_{l}}, b_{i_{1}}\right)\right)>0
$$

or equivalently,

$$
\left.\min \left(R^{A}\left(a_{i_{1}}, a_{i_{2}}\right)\right), R^{A}\left(a_{i_{2}}, a_{i_{3}}\right), \ldots, R^{A}\left(a_{i_{l}}, a_{i_{1}}\right)\right)>0,
$$

which contradicts the fact that $R^{A}$ is min-acyclic.

Thus $\bar{R}^{T}$ is a compatible extension of $R$, and it is min-transitive because it is a transitive closure. Let us prove that $\bar{P}^{T}\left(b_{i}, a_{i}\right)=1$ for all $i \in\{1, \ldots, n\}$. Fix an arbitrary $i \in\{1, \ldots, n\}$. Since $\bar{R}^{T}\left(b_{i}, a_{i}\right) \geq \bar{R}\left(b_{i}, a_{i}\right)=1$, it only remains to prove that $\bar{R}^{T}\left(a_{i}, b_{i}\right)=0$. It is clear that $R\left(a_{i}, b_{i}\right)=0$, otherwise $R^{A}\left(a_{i}, a_{i}\right)>0$ against the fact that $R^{A}$ is min-acyclic. Furthermore $\bar{R}\left(a_{i}, b_{i}\right)=0$, since otherwise $R\left(a_{i}, b_{i}\right) \neq \bar{R}\left(a_{i}, b_{i}\right)$ thus necessarily $\left(a_{i}, b_{i}\right)=\left(b_{j}, a_{j}\right)$ for some $j$, and because $R$ is reflexive

$$
\begin{aligned}
& 1=R\left(a_{i}, a_{i}\right)=R\left(a_{i}, b_{j}\right)=R^{A}\left(a_{i}, a_{j}\right), \\
& 1=R\left(a_{j}, a_{j}\right)=R\left(a_{j}, b_{i}\right)=R^{A}\left(a_{j}, a_{i}\right),
\end{aligned}
$$


against the fact that $R^{A}$ is min-acyclic.

Now, if $\bar{R}^{T}\left(a_{i}, b_{i}\right)>0$ then there must be elements $c_{1}, \ldots, c_{k}$ such that

$$
\min \left(\bar{R}\left(a_{i}, c_{1}\right), \ldots, \bar{R}\left(c_{k}, b_{i}\right)\right)>0 .
$$

So the Claim above applies again. Eq. (4) contradicts $R\left(a_{i}, b_{i}\right)=0$. If Eq. (5) applies, then there exists a set $\left\{j_{1}, \ldots, j_{l}\right\} \in\{1, \ldots, n\}$ such that:

$$
\min \left(R\left(a_{i}, b_{j_{1}}\right), R\left(a_{j_{1}}, b_{j_{2}}\right), \ldots, R\left(a_{j_{l}}, b_{i}\right)\right)>0 .
$$

Equivalently,

$$
\min \left(R^{A}\left(a_{i}, a_{j_{1}}\right), R^{A}\left(a_{j_{1}}, a_{j_{2}}\right), \ldots, R^{A}\left(a_{j_{l}}, a_{i}\right)\right)>0 .
$$

This is a cycle in $R^{A}$, which is absurd.

From $\bar{R}^{T}\left(a_{i}, b_{i}\right)=0$ and $\bar{R}^{T}\left(b_{i}, a_{i}\right)=1$, one has $\bar{P}^{T}\left(b_{i}, a_{i}\right)=1-i(1,0)=1$.

Since $\bar{R}^{T}$ is transitive and reflexive, Theorem 3 assures that there exists a total order $\tilde{R}$ that is a compatible extension of $\bar{R}^{T}$. Then $\tilde{P}\left(b_{i}, a_{i}\right)=1$ for all $i \in\{1, \ldots, n\}$. Furthermore, because $\tilde{R}$ is a compatible extension of $\bar{R}^{T}$ and this is a compatible extension of $R$, then $\tilde{R}$ is a compatible extension of $R$, which completes the proof.

Now we discuss to what extent the necessary and sufficient conditions in [4, Theorem 2] can be generalized to the context of strict preference relations obtained by generators. Our contribution in this setting is Theorem 5 below, which provides a sufficient condition and refers to the generator min. Then we show that the only generator for which the result holds true is the minimum. Finally, we check that our sufficient condition is not necessary.

We first extend Definitions 7, 8 and 9 in order to fit the relevant statement.

Definition 17. The relation $R^{I}$ associated with $R$ and $X_{I}$ is defined as follows:

$$
R^{I}(a, b)=R(a, \delta(b)), \text { for all } a, b \in X_{I},
$$

where $\delta(b)$ is the function introduced in Definition 6.

Definition 18. We say that $R^{I}$ is $\delta$-min-cyclic along $X_{I}^{n}$ if

$$
\begin{aligned}
& \min \left(R^{I}\left(a_{1}, a_{2}\right), R^{I}\left(a_{2}, a_{3}\right), \ldots, R^{I}\left(a_{k}, a_{1}\right)\right) \\
& \quad=\min \left(R^{I}\left(\delta\left(a_{1}\right), \delta\left(a_{k}\right)\right), \ldots R^{I}\left(\delta\left(a_{2}\right), \delta\left(a_{1}\right)\right)\right), \text { for all } a_{1}, \ldots, a_{k} \in X_{I}^{n} .
\end{aligned}
$$

Definition 19. The relation $R^{G}$ associated with $R, n \leq p$ and $X_{I}$ is defined by

$$
\begin{aligned}
R^{G}\left(a_{i}, a_{j}\right)=\max & \left(R\left(a_{i}, b_{j}\right),\right. \\
& \left.\sup _{\left\{c_{1}, \ldots c_{k}\right\} \subseteq X_{I}^{n}}\left(\min \left(R\left(a_{i}, \delta\left(c_{1}\right)\right), R\left(c_{1}, \delta\left(c_{2}\right)\right), \ldots, R\left(c_{k}, b_{j}\right)\right)\right)\right) .
\end{aligned}
$$

Definition 20. The relation $R^{G}$ is $\delta$-consistent with $X_{I}$ and $n \leq p$ if $\min \left(R^{G}\left(a_{i_{1}}, a_{i_{2}}\right), \ldots, R^{G}\left(a_{i_{k}}, a_{i_{1}}\right)\right)>0$ implies $i_{t}>n$ for all $t \in\{1, \ldots, k\}$. 
Theorem 5. Let us assume that all indifference and strict preference relations are derived by Eq. (3) via the generator min.

Let $X_{I}=\left\{a_{1}, \ldots, a_{p}, b_{1}, \ldots, b_{p}\right\}$ be an ordered list of possibly repeated elements of $X$ and let $n \leq p$. Let $R$ be a fuzzy preorder on $X$.

If the following properties hold true:

a) the relation $R^{G}$ is $\delta$-consistent with $X_{I}$ and $n$, and then

b) $R^{I}$ is $\delta$-min-cyclic along $X_{I}^{n}$,

c) there exists a fuzzy order $\tilde{R}$ that is a compatible extension of $R$ such that $\tilde{P}\left(b_{i}, a_{i}\right)=1$ for each $i=1, \ldots, n$ and $\tilde{I}\left(b_{i}, a_{i}\right)=1$ for $i=n+1, \ldots, p$, where $\tilde{P}$ and $\tilde{I}$ are the strict preference and indifference relations obtained from $\tilde{R}$.

Proof. We use the properties of the auxiliary relation $\bar{R}$ defined as follows:

$$
\begin{aligned}
\bar{R}(a, b) & =\max (R(a, b), \\
& \left.\sup _{\left\{d_{1}, \ldots d_{k}\right\} \subseteq X_{I}^{n}}\left(\min \left(R\left(a, \delta\left(d_{1}\right)\right), R^{I}\left(d_{1}, d_{2}\right), \ldots, R^{I}\left(d_{k-1}, d_{k}\right), R\left(d_{k}, b\right)\right)\right)\right) .
\end{aligned}
$$

Then $\bar{R}\left(a_{i}, b_{j}\right)=R^{G}\left(a_{i}, a_{j}\right)$ for all $i, j$.

It is easy to check that $\bar{R}$ is reflexive and min-transitive. Let $\bar{I}$ be the symmetric part of $\bar{R}$, then the fact that $\bar{I}\left(a_{i}, b_{i}\right)=1$ for all $i=n+1, \ldots, p$ follows from

$$
\begin{aligned}
& \bar{R}\left(a_{i}, b_{i}\right) \geq \min \left(R\left(a_{i}, \delta\left(b_{i}\right)\right), R\left(b_{i}, b_{i}\right)\right)=1, \quad \text { for each } i, \\
& \bar{R}\left(b_{i}, a_{i}\right) \geq \min \left(R\left(b_{i}, \delta\left(a_{i}\right)\right), R\left(a_{i}, a_{i}\right)\right)=1, \quad \text { for each } i .
\end{aligned}
$$

Furthermore we claim that $\bar{R}$ extends $R$. It is clear that $R \subseteq \bar{R}$. Let $\bar{P}$ denote the asymmetric component of $\bar{R}$. To prove $P \subseteq \bar{P}$ we fix arbitrary $a, b \in X$, then we need to assure $\bar{P}(a, b) \geq P(a, b)$. Two cases arise. If $\bar{R}(b, a)=R(b, a)$ then $\bar{P}(a, b)=\bar{R}(a, b)-\min (\bar{R}(a, b), \bar{R}(b, a)) \geq R(a, b)-\min (R(a, b), R(b, a))=$ $P(a, b)$ and we are done. If $\bar{R}(b, a)>R(b, a)$ then we get a contradiction under the assumption $P(a, b)>\bar{P}(a, b)$ as follows. Observe that now since $P(a, b)>0$, $R(a, b)>R(b, a)$ and $\bar{R}(a, b)>0$ hold true. From $\bar{R}(b, a)>R(b, a)$ we deduce the existence of $d_{1}, \ldots, d_{k} \in X_{I}^{n}$ such that

$$
\min \left(R^{I}\left(d_{1}, d_{2}\right), \ldots, R^{I}\left(d_{k-1}, d_{k}\right)\right)>R(b, a)
$$

and also $R\left(b, \delta\left(d_{1}\right)\right)>R(b, a)$ and $R\left(d_{k}, a\right)>R(b, a)$. Because $R(a, b)>R(b, a)$, $\min \left(R\left(d_{k}, a\right), R(a, b), R\left(b, \delta\left(d_{1}\right)\right)\right)>R(b, a)$ which implies $R\left(d_{k}, \delta\left(d_{1}\right)\right)>R(b, a)$. Hence $\min \left(R^{I}\left(d_{1}, d_{2}\right), R^{I}\left(d_{2}, d_{3}\right), \ldots, R^{I}\left(d_{k}, d_{1}\right)\right)>R(b, a)$. Therefore, the $\delta$ min-cyclicity of $R^{I}$ produces

$$
\min \left(R^{I}\left(\delta\left(d_{1}\right), \delta\left(d_{k}\right)\right), \ldots, R^{I}\left(\delta\left(d_{2}\right), \delta\left(d_{1}\right)\right)\right)>R(b, a) .
$$

Then because

$$
R(b, a) \geq \min \left(R\left(b, \delta\left(d_{1}\right)\right), R\left(\delta\left(d_{1}\right), d_{k}\right), R\left(d_{k}, a\right)\right)>R(b, a),
$$

we get a contradiction that completes the argument. 
Now we are ready to conclude. If $n=0$ then any fuzzy order $\tilde{R}$ that is a compatible extension of $\bar{R}$ is a compatible extension of $R$ too. If $n>0$ then we can apply Theorem 4: the relation $\bar{R}^{A}$ is min-acyclic on ${ }^{n} X_{I}=\left\{a_{1}, \ldots, a_{n}, b_{1}, \ldots, b_{n}\right\}$ since $R^{G}$ is $\delta$-consistent. Therefore there exists an order $\tilde{R}$ that extends $\bar{R}$ such that $\tilde{P}\left(b_{i}, a_{i}\right)=1$ for $i \leq n$. In addition to this, $\tilde{I}\left(b_{i}, a_{i}\right)=1$ for all $i \geq n+1$ because $\tilde{R}$ extends $\bar{R}$ and $\bar{I}\left(a_{i}, b_{i}\right)=1$ for all $i \geq n+1$. This finishes the proof.

The following example proves that the restriction to the generator min in the statement of Theorem 5 is not superfluous:

Example 2. Fix a generator $i$ other than the minimum. Then there exists a value $x$ such that $i(x, x)<x=\min (x, x)$. Consider the weak preference relation $R$ defined on $X=\left\{c, a_{1}, b_{1}, a_{2}, b_{2}, d\right\}$ as follows:

\begin{tabular}{c|cccccc}
$R$ & $c$ & $a_{1}$ & $b_{1}$ & $a_{2}$ & $b_{2}$ & $d$ \\
\hline$c$ & 1 & $x$ & 0 & 0 & $x$ & $x$ \\
$a_{1}$ & $x$ & 1 & 0 & 0 & $x$ & $x$ \\
$b_{1}$ & 0 & 0 & 1 & 1 & 0 & 0 \\
$a_{2}$ & 0 & 0 & $x$ & 1 & 0 & 0 \\
$b_{2}$ & 1 & $x$ & 0 & 0 & 1 & $x$ \\
$d$ & $x$ & 1 & 0 & 0 & $x$ & 1
\end{tabular}

This relation is min-transitive. Now consider the set $X_{I}=\left\{a_{1}, a_{2}, b_{1}, b_{2}\right\}$.

We are interested in imposing the conditions

$$
\tilde{I}\left(a_{1}, b_{1}\right)=1 \text { and } \tilde{I}\left(a_{2}, b_{2}\right)=1
$$

on compatible fuzzy order extensions. Observe that $R^{G}$ is trivially $\delta$-consistent with $X_{I}$ and $n$ since $n=0$. It can also be checked that $R^{I}$ is $\delta$-min-cyclic. However, if we use the generator $i$ to derive strict preference from weak preference relations then we claim that there exists no fuzzy order $\tilde{R}$ that extends $R$ and verifies the conditions in Eq. (7).

To prove our claim, observe that

$$
P(c, d)=R(c, d)-i(R(c, d), R(d, c))=x-i(x, x)>0 .
$$

Provided $\tilde{R}$ extends $R, \tilde{R}(a, b) \geq R(a, b)$ for all $\{a, b\} \subseteq X$. Since $\tilde{R}$ satisfies the conditions in Eq. (7) and is min-transitive we obtain

$$
\begin{aligned}
\tilde{R}(d, c) & \geq \min \left(\tilde{R}\left(d, a_{1}\right), \tilde{R}\left(a_{1}, b_{1}\right), \tilde{R}\left(b_{1}, a_{2}\right), \tilde{R}\left(a_{2}, b_{2}\right), \tilde{R}\left(b_{2}, c\right)\right) \\
& =\min (1,1,1,1,1)=1 .
\end{aligned}
$$

Hence $\tilde{P}(c, d)=\tilde{R}(c, d)-i(\tilde{R}(c, d), \tilde{R}(d, c))=\tilde{R}(c, d)-i(\tilde{R}(c, d), 1)=0$, which proves $P \nsubseteq \tilde{P}$.

Now we proceed to prove that $\delta$-consistency of $R^{G}$ is a necessary condition if we want to extend a preorder as in Theorem 5 , in the following terms: 
Proposition 2. Under the assumptions of Theorem 5, condition c) implies condition a).

Proof. Observe that because either $\tilde{P}\left(b_{i}, a_{i}\right)=1$ or $\tilde{I}\left(b_{i}, a_{i}\right)=1$ for all $i \in$ $\{1, \ldots, p\}$, it must be the case that $\tilde{R}\left(b_{i}, a_{i}\right)=1$ for all $i \in\{1, \ldots, p\}$.

We argue by contradiction. Let us suppose that

$\min \left(R^{G}\left(a_{i_{1}}, a_{i_{2}}\right), \ldots, R^{G}\left(a_{i_{k}}, a_{i_{1}}\right)\right)>0$, with $i_{t} \leq n \quad$ for some $t \in\{1, \ldots, k\}$.

Then $\tilde{P}\left(b_{i_{t}}, a_{i_{t}}\right)=1$ and $R^{G}\left(a_{i_{j}}, a_{i_{j+1}}\right)>0$ for all $j \in\{1, \ldots, k\}$ (along this proof we use the convention $k+1=1$ ). From the definition of $R^{G}$ we deduce that one of the following cases must hold:

1. $R\left(a_{i_{j}}, b_{i_{j+1}}\right)>0$, therefore $\tilde{R}\left(a_{i_{j}}, b_{i_{j+1}}\right)>0$, for all $j \in\{1, \ldots, k\}$. By hypothesis $\tilde{R}\left(b_{i_{j}}, a_{i_{j}}\right)>0$ for all $j \in\{1, \ldots, k\}$. Then, since $\tilde{R}$ is min-transitive, $\tilde{R}\left(a_{i_{t}}, b_{i_{t}}\right)>0$. Also, $\tilde{P}\left(b_{i_{t}}, a_{i_{t}}\right)=1$ by hypothesis since $i_{t} \leq n$, a contradiction.

2. Assume $\min \left(R\left(a_{i_{j}}, \delta\left(c_{1}\right)\right), R\left(c_{1}, \delta\left(c_{2}\right)\right), \ldots, R\left(c_{l}, b_{i_{j+1}}\right)\right)>0$ for some $j$. This implies

$$
\min \left(\tilde{R}\left(a_{i_{j}}, \delta\left(c_{1}\right)\right), \tilde{R}\left(c_{1}, \delta\left(c_{2}\right)\right), \ldots, \tilde{R}\left(c_{l}, b_{i_{j+1}}\right)\right)>0,
$$

where $c_{1}, \ldots, c_{l} \in X_{I}^{n}$. Then by hypothesis

$$
\tilde{I}\left(c_{i}, \delta\left(c_{i}\right)\right)=1, \quad \forall i \in\{1, \ldots, l\} \text { since they are in } X_{I}^{n} .
$$

From this fact and the previous inequality we deduce

$$
\min \left(\tilde{R}\left(a_{i_{j}}, \delta\left(c_{1}\right)\right), \tilde{I}\left(\delta\left(c_{1}\right), c_{1}\right), \tilde{R}\left(c_{1}, \delta\left(c_{2}\right)\right), \ldots, \tilde{I}\left(\delta\left(c_{l}\right), c_{l}\right), \tilde{R}\left(c_{l}, b_{i_{j+1}}\right)\right)>0 .
$$

Since $\tilde{R}$ is min-transitive, we also deduce $\tilde{R}\left(a_{i_{j}}, b_{i_{j+1}}\right)>0$ for every $j \in$ $\{1, \ldots, k\}$. By hypothesis $\tilde{R}\left(b_{i}, a_{i}\right)=1$ for all $i$, therefore by min-transitivity we get $\tilde{R}\left(a_{i_{t}}, b_{i_{t}}\right)>0$, contradicting the assumption $\tilde{P}\left(b_{i_{t}}, a_{i_{t}}\right)=1$.

So in either case we obtain a contradiction.

However, Theorem 5 does not provide a complete characterization since condition $b$ ) is not implied by $c$ ) as we next show.

Example 3. Let $X=\left\{a_{1}, b_{1}, a_{2}, b_{2}\right\}$ and define the following fuzzy relations $R$ and $\tilde{R}$ on $X$ as follows:

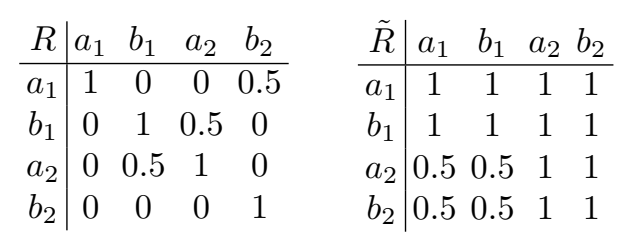

Then $R$ is a fuzzy preorder, $\tilde{R}$ is a fuzzy order, and for $X_{I}=X$ and $n=0$, the relation $\tilde{R}$ is a compatible extension of $R$ such that $\tilde{I}\left(b_{1}, a_{1}\right)=1$ and $\tilde{I}\left(b_{2}, a_{2}\right)=1$. 
We claim that $R^{I}$ is not $\delta$-min-cyclic. To check it, consider the alternatives $a_{1}$ and $a_{2}$. One has $\min \left(R^{I}\left(a_{1}, a_{2}\right), R^{I}\left(a_{2}, a_{1}\right)\right)>0$ because $R^{I}\left(a_{1}, a_{2}\right)=R\left(a_{1}, b_{2}\right)>0$ and $R^{I}\left(a_{2}, a_{1}\right)=R\left(a_{2}, b_{1}\right)>0$, whereas

$$
\begin{aligned}
& \min \left(R^{I}\left(\delta\left(a_{1}\right), \delta\left(a_{2}\right)\right), R^{I}\left(\delta\left(a_{2}\right), \delta\left(a_{1}\right)\right)\right)=R^{I}\left(\delta\left(a_{2}\right), \delta\left(a_{1}\right)\right)=R\left(\delta\left(a_{2}\right), a_{1}\right) \\
& \quad=R\left(b_{2}, a_{1}\right)=0 .
\end{aligned}
$$

\section{Concluding remarks and further research}

In this contribution we have studied the problem of extending fuzzy preorders to fuzzy orders, with special attention to the case when additional conditions on pairwise comparisons by strict preference and indifference are imposed. The concept under inspection -compatible extensions- depends on the construction of associated strict preference relations. We have focused on the most succesful proposal in this regard, namely, the use of generators.

Our results prove that 1-Lipschitz generators, and particularly the minimum t-norm, are especially suitable to produce results on extensions of fuzzy preorders. They permit to expand crisp approaches to this analysis in a natural way. A predictable question for further research is if other types of generators also suffice to prove analogous statements.

Another line of inspection starts with different constructions of associated strict preference relations. For example, the decomposition of weak preference relations in Georgescu [25] can be investigated instead. Now one can guess that results more similar to the inspiring crisp characterization theorems can be proven. The reason is that Georgescu's construction is much closer to the crisp spirit than the construction from generators.

We expect to produce results in these lines in the future.

\section{Acknowledgement}

This work has been partially supported by the Research Project ECO2012-31933 (Spanish Ministerio de Economía y Competitividad).

The authors gratefully acknowledge constructive comments by the participants at EUROFUSE2013, especially Bernard de Baets, who suggested to investigate the topic from the perspective of generators. The valuable suggestions of two anonymous referees have also contributed to improve the manuscript.

\section{References}

1. J. C. R. Alcantud. Topological properties of spaces ordered by preferences. International Journal of Mathematics and Mathematical Sciences, 22, 17-27 (1999).

2. J. C. R. Alcantud. Orderability of topological spaces by continuous preferences. International Journal of Mathematics and Mathematical Sciences, 27, 505-512 (2001).

3. J. C. R. Alcantud. Characterization of the existence of maximal elements of acyclic relations. Economic Theory, 19, 407-416 (2002). 
4. J. C. R. Alcantud. Conditional ordering extensions. Economic Theory, 39, 495-503 (2009).

5. K. J. Arrow. Social Choice and Individual Values, 2nd ed. John Wiley \& Sons, 1963.

6. S. Bade. Nash equilibrium in games with incomplete preferences. Economic Theory, 26, 309-332 (2005).

7. U. Bodenhofer, F. Klawonn. A formal study of linearity axioms for fuzzy orderings. Fuzzy Sets and Systems, 145, 323-354 (2004).

8. G. Bosi, G. Herden. On a strong continuous analogue of the Szpilrajn theorem and its strengthening by Dushnik and Miller. Order, 22, 329-342 (2005).

9. G. Bosi, G. Herden. On a possible continuous analogue of the Szpilrajn theorem and its strengthening by Dushnik and Miller. Order, 23, 271-296 (2006).

10. W. Bossert. Intersection quasi-orderings: An alternative proof. Order, 16, 221-225 (1999).

11. W. Bossert, Y. Sprumont, K. Suzumura. Upper semicontinuous extensions of binary relations. Journal of Mathematical Economics, 37, 231-246 (2002).

12. De Baets, B., Fodor, J.: Additive fuzzy preference structures: the next generation. In: De Baets B., Fodor, J. (eds.) Principles of Fuzzy Preference Modelling and Decision Making, pp. 15-25. Academia Press (2003)

13. B. De Baets, J. Fodor. Twenty years of fuzzy preference structures (1978-1997). JORBEL, 37, 61-82 (1997).

14. M. J. Campión, J. C. Candeal, E. Induráin. On Yi's extension property for totally preordered topological spaces. Journal of the Korean Mathematical Society, 43, 159-181 (2006).

15. N. Caspard, B. Leclerc, B. Monjardet. Ensembles ordonnés finis: concepts, résultats et usages. Springer, 2007.

16. C. Chambers, B. Yenmez. Choice and matching. Available at SSRN 2291045 (2013).

17. D. Donaldson, J. A. Weymark. A quasiordering is the intersection of orderings. Journal of Economic Theory, 78, 382-387 (1998).

18. R.G. Downey. Computability theory and linear orderings. In: Y. L. Ershov et al. (eds.) Handbook of Recursive Mathematics, vol. 2, pp. 823-976. North Holland, Amsterdam (1998).

19. J. Duggan. A general extension theorem for binary relations. Journal of Economic Theory, 86, 1-16 (1999).

20. B. Dushnik, E. W. Miller. Partially ordered sets. American Journal of Mathematics, 63, 600-610 (1941).

21. J. Fodor. An axiomatic approach to fuzzy preference modelling. Fuzzy Sets and Systems, 52, 47-52 (1992).

22. J. Fodor, M. Roubens: Valued preference structures. European Journal of Operational Research, 79, 277-286 (1994).

23. J. Fodor, M. Roubens: Fuzzy Preference Modelling and Multicriteria Decision Support. Kluwer Academic Publishers, Dordrecht (1994).

24. S. Foldes, J. Szigeti. Maximal compatible extensions of partial orders. Journal of the Australian Mathematical Society, 81, 245-252 (2006).

25. I. Georgescu. Compatible extensions of fuzzy relations. Journal of Systems Science and Systems Engineering, 12, 332-349 (2003).

26. I. Georgescu. Fuzzy Choice Functions: A Revealed Preference Approach. Springer, 2007.

27. I. Georgescu. Compatible Extensions and Consistent Rationalizability: A Fuzzy Approach. TUCS Technical Reports 881, Turku Centre for Computer Science, 2008. 
28. S. Gottwald. Fuzzy Sets and Fuzzy Logic. Vieweg, 1993.

29. B. Hansson, Choice structures and preference relations. Synthese, 18, 443-458 (1968).

30. G. Herden, A. Pallack. On the continuous analogue of the Szpilrajn Theorem I. Mathematical Social Sciences, 43, 115-134 (2002).

31. U. Höhle, N. Blanchard. Partial ordering in L-underdeterminate sets. Information Sciences, 35, 133-144 (1985).

32. J.-Y. Jaffray. Semicontinuous extension of a partial order. Journal of Mathematical Economics, 2, 395-406 (1975).

33. E. P. Klement, R. Mesiar, E. Pap, Triangular Norms, 386 pages, Kluwer Academic Publishers, Boston/London/Dordrecht, (2000).

34. S. Lahiri. A simple proof of Suzumuras extension theorem for finite domains with applications. Journal of Applied Mathematics and Decision Sciences, 6, 183-190 (2002).

35. A. Laruelle, F. Valenciano. Quaternary dichotomous voting rules. Social Choice and Welfare, 38, 431-454 (2012).

36. M. Mabrouk. On the extension of a preorder under translation invariance. MPRA Paper No. 15407 (2009).

37. I. Montes, S. Díaz, S. Montes. On complete fuzzy preorders and their characterizations. Soft Computing, 15, 1999-2011 (2010).

38. K. Nehring, C. Puppe. Extended partial orders: a unifying structure for abstract choice theory. Annals of Operations Research, 80, 27-48 (1998).

39. S. A. Orlovsky, Decision-making with a fuzzy preference relation. Fuzzy Sets and Systems, 1, 155-167 (1978).

40. S. V. Ovchinnikov, M. Roubens. On fuzzy strict preference, indifference and incomparability relations. Fuzzy Sets and Systems, 47, 313-318 (1992).

41. M. K. Richter. Revealed preference theory. Econometrica, 34, 635-645 (1966).

42. D.K. Roy. Effective extensions of partial orders. Mathematical Logic Quarterly, 36, 233-236 (2000).

43. L. A. Sholomov. Explicit form of neutral decision rules for basic rationality conditions. Mathematical Social Sciences, 39, 81-107 (2000).

44. K. Suzumura. Remarks on the theory of collective choice. Economica, 43, 381-390 (1976).

45. K. Suzumura. Rational Choice, Collective Decisions, and Social Welfare. Cambridge University Press, 1983.

46. L.-G. Svensson. Equity among generations. Econometrica, 48, 1251-1256 (1980).

47. E. Szpilrajn. Sur l'éxtension de l'ordre partiel. Fundamenta Mathematicae, 16, 386-389 (1930).

48. J.E. Weymark. A generalization of Moulin's Pareto extension theorem, Mathematical Social Sciences, 39, pp. 235-240 (2000).

49. G. Yi. Continuous extension of preferences. Journal of Mathematical Economics, 22, 547-555 (1993).

50. L. Zadeh. Fuzzy sets. Information and Control, 3, 338-353 (1965).

51. L. Zadeh. Similarity relations and fuzzy orderings. Information Sciences, 3, 177200 (1971). 